\title{
UROLOGIC LAPAROSCOPY WITH A NEW BLUNT- TIPPED TROCAR: SAFE, RAPID ACCESS WITHOUT THE USE OF FASCIAL SUTURES
}

\author{
DANA A. OHL, M.D. \\ GARY J. FAERBER, M.D. \\ WILLIAM W. HURD, M.D.*
}

From the Section of Urology, Department of Surgery, Department of Gynecology and Obstetrics, University of Michigan Medical Center; and Section of Urology, Department of Surgery, Veteran's Affairs Medical Center, Ann Arbor, Michigan

\begin{abstract}
Fifteen patients underwent successful laparoscopic procedures where a new blunt-tipped trocar was used both to gain initial access to and to insufflate the abdominal cavity. The use of this new trocar obviates the need for the use of the Veress needle and appears to be both time-saving and possibly safer. Unlike the Hasson-type trocar, the blunt trocar technique does not require the use of fascial sutures for prevention of air leaks.
\end{abstract}

In recent years there has been a marked increase in the use of laparoscopy for urologic indications. New procedures are continually being brought to clinical use. Most urologic laparoscopic procedures involve entry into and insufflation of the peritoneal cavity for access to the surgical site. The Veress needle is commonly used to puncture and insufflate the abdomen and sharp-tipped trocars penetrate into the insufflated abdomen to allow entry of the laparoscope and surgical instruments.

Unfortunately, placement of the Veress needle and primary trocar are blind procedures, with the risk of penetration of a viscus or major blood vessel. Indeed, many of the serious complications from laparoscopy come from these maneuvers. We report our initial experience with a new blunttipped trocar for initial access in urologic laparoscopy, which combines the advantages of fast and simple abdominal access seen in sharp-tipped trocars and the safety of an open placement technique.

\section{MATERIAL AND METHODS}

From January 1993 to July 1993, 15 patients (14 male, 1 female) underwent a urologic laparoscopic procedure in which initial access to the peritoneal

${ }^{\star} \mathrm{Dr}$. Hurd is a Consultant for Apple Medical Corporation. Submitted: December 7, 1993, accepted (with revisions): January 6, 1994 cavity was achieved utilizing the blunt-tipped trocar (Hurd trocar, Apple Medical Corporation, Bolton, MA; Fig. 1). The $10 \mathrm{~mm}$ sleeve has threads on the outside surface to stabilize the device in the fascia. The air seal is designed for $10 \mathrm{~mm}$ instruments with a $5 \mathrm{~mm}$ reducer for smaller instruments. An additional small seal is supplied to cover the insufflation port if the device is used as a secondary trocar. The trocar, which is inserted through the sheath during placement into the abdomen, is blunt-tipped for safety, but conical in shape to allow fascial dilation and a tight fascial seal during open insertion.

Six men underwent bilateral varicocele ligation and 6 had a unilateral varicocele ligation, 2 under. went pelvic lymph node dissection for staging of prostatic carcinoma, and the one woman in the series underwent renal cyst decortication. Patient ages averaged thirty-six years ( 14 to 78 years). Mean weight was $76 \mathrm{~kg}$ (range 44 to 95 ) and height was $163 \mathrm{~cm}$ (range 144 to 193). Three patients had undergone previous abdominal operations (one bilateral herniorrhaphy, one unilateral herniorraphy, one open cholecystectomy).

The method of primary trocar placement is as follows: After induction of general endotracheal anesthesia, a standard abdominal shave and preparation is performed and an indwelling urinary catheter is placed in the bladder. A 10 to $15 \mathrm{~mm}$ infraumbilical incision is made and the rectus 

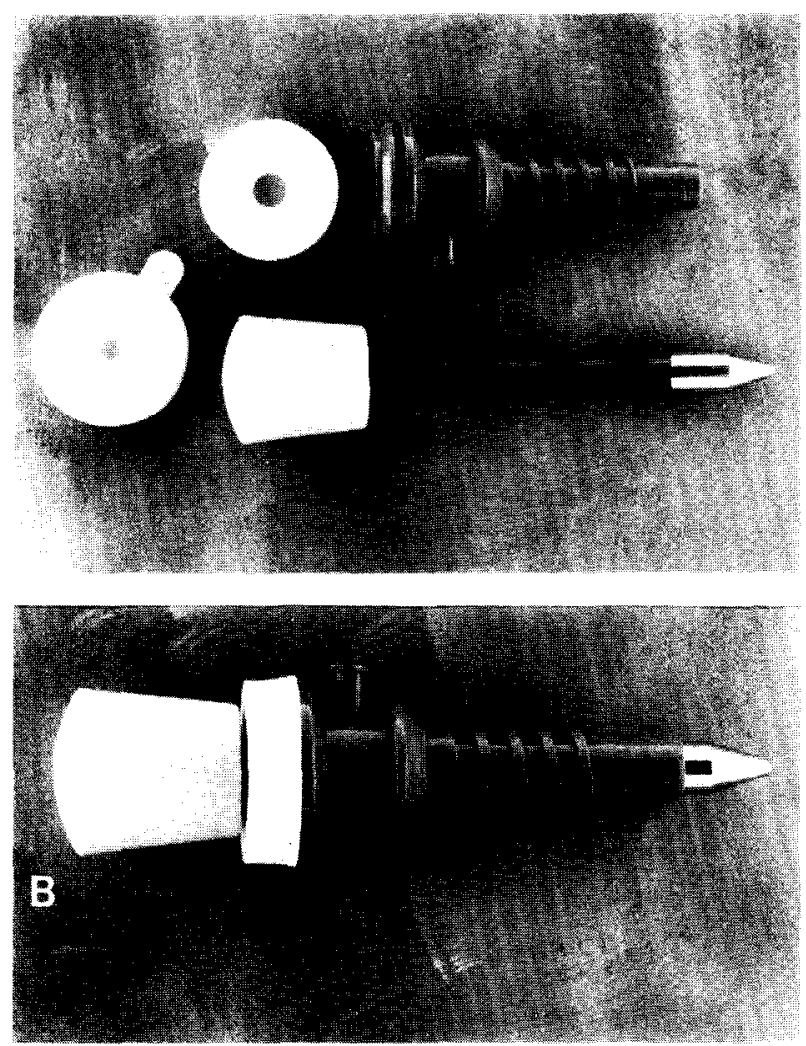

FIGURE 1. Trocar used in this study. (A) The disassembled components of the trocar. See text for explanation of components. (B) The trocar is assembled, ready for use.

abdominus fascia identified by blunt dissection with Crile artery forceps. Kocher clamps are applied to the fascia bilaterally and the abdominal wall is elevated off the intra-abdominal contents. A vertical $5 \mathrm{~mm}$ incision is made into the fascia and the Crile artery forceps used to bluntly puncture the peritoneal cavity at the insertion of the umbilicus. The forceps are directed toward the patient's head to take advantage of the close proximity of the peritoneum at the umbilicus. The peritoneal opening is dilated to 5 to $6 \mathrm{~mm}$ with the hemostat and the trocar advanced into the abdomen, again directed toward the patient's head. The fascia is held in an elevated position during the entire insertion procedure.

Proper placement of the trocar into the peritoneal cavity is confirmed by visual inspection with the $10 \mathrm{~mm}$ laparoscope. The trocar is then secured to the abdominal wall with the use of the threaded outer sleeve. Insufflation is performed through the trocar itself. Additional trocars necessary for the procedures are placed into the abdominal wall under visual control.

Heights and weights were recorded for all patients. Body mass index was calculated with the

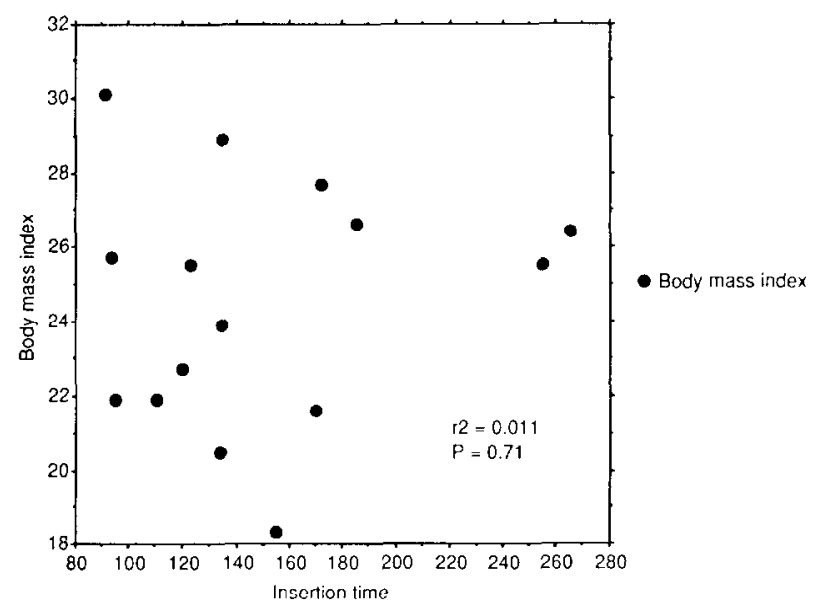

FIGURE 2. Comparison of body mass index and insertion time (in seconds). There is no significant correlation.

formula: (height in meters) ${ }^{2} \times$ weight $(\mathrm{kg})=$ body mass index. Physical characteristics of the patients were compared to time of trocar insertion and time of insufflation with linear regression analysis. A $p$ value of $<0.05$ was considered significant.

\section{RESULTS}

Average overall operating time was one hundred fifteen minutes (range 45 to 265 minutes). The average skin incision length was $13 \mathrm{~mm}$. The average time interval from the infraumbilical incision to visual confirmation of the trocar within the peritoneal cavity was one hundred forty-nine seconds (95 to 265 seconds). Time to full insufflation was an additional eighty-two seconds (range 46 to 115 seconds).

There were no complications from the surgical procedures and, specifically, none resulting from the primary trocar insertion. No air leaks were seen in any patient, verifying adequate peritoneal seal around the trocar.

In comparing the body habitus of the patients to the insertion time of the trocar and time to full insufflation, no correlation could be found. The comparisons between patient body mass index and insertion time is noted in Figure 2. There was also no correlation between insertion time and patient sequence number in the series.

\section{COMMENT}

Laparoscopy has proven to be a safe surgical technology. Urological applications of laparoscopy include varicocele ligation, staging pelvic lymphadenectomy, nephrectomy, marsupialization of renal cysts, locating the nonpalpable testes, drainage of lymphoceles, and retroperitoneal needle suspension urethropexy. ${ }^{1-7}$ The list of indications for urologic laparoscopy is progressively 
increasing. Clearly, laparoscopy has surpassed the pure diagnostic procedure category and in some instances has clearly replaced "standard" open surgery as the treatment of choice. ${ }^{8}$

During laparoscopy, the most likely causes of a serious complication are improper placement of a Veress needle or sharp-tipped trocar, resulting in vascular or bowel injury.9-11 Despite many recent advances in laparoscopic equipment, there has been little modification of the Veress needle technique most often used in creating a pneumoperitoneum. The Veress needle has a spring-loaded blunt obturator designed to protect the intra-abdominal contents. Theoretically, the obturator springs out to protect intra-abdominal structures after entering the peritoneum, but the narrow tip (approximately 14 gauge) still has the capability of perforating fixed structures, such as adhesed bowel or retroperitoneal vessels. Despite the apparent safety features of the Veress needle, bowel and vascular injury, and inadvertent insufflation of structures other than the peritoneal cavity have been reported. ${ }^{12}$

The incidence of major injuries during laparoscopy has varied in reports in the literature. One of the largest series concentrating on Veress needle and trocar injuries was the survey of Yuzpe. ${ }^{10}$ Four hundred seven gynecologists responded to a survey on the topic, and one quarter of them had experienced at least one injury related to the needle or trocar insertion. The report does not allow incidence figures to be calculated because multiple injuries by the same surgeons were not noted, but in the series, one hundred nine surgeons reported pneumoperitoneum needle injury and one hundred four reported a major organ injury by the primary trocar. ${ }^{10}$

A German series of gynecologic laparoscopy gave more detailed information. The incidence of injury to blood vessels was 0.178 per 1,000 cases from Veress needle insertion and 0.374 per 1,000 cases from the primary trocar insertion in procedures performed by clinics. Private practices reported slightly higher rates of injury of 0.22 per 1,000 cases and 0.611 per 1,000 cases, respectively. ${ }^{13}$

Deziel et al. ${ }^{14}$ reported on complication rates from 77,604 laparoscopic cholecystectomies. There were one hundred nine bowel injuries $(0.14 \%)$. These were not separated by mechanism of injury, but are stated to have included Veress needle insertion, trocar placement, cautery burns, and retraction injury. Vascular injuries were seen in 0.25 percent of cases, with the Veress needle or trocar responsible for all retroperitoneal vessel injuries $(0.05 \%$ incidence) and some of the injuries to "other intra-abdominal vessels" ( $0.05 \%$ incidence). Perhaps this large survey of general surgery cases may predict results that would be seen in a large survey of urologic laparoscopy, since laparoscopic surgery is relatively new to both specialities.

The need for more control during initial access to the peritoneal cavity led Hasson ${ }^{15}$ to develop a cannula and open insertion technique that obviated the need for the Veress needle and blind insertion with a sharp trocar. The blunt-tipped Hasson trocar cannula is inserted through a small incision into the peritoneal cavity and a coneshaped sleeve is brought down tightly against the peritoneotomy and secured by fascial sutures tied to the arms of the cannula. This technique was an extremely important advance in the safety of laparoscopy, but the need for placement of fascial sutures, difficulty with maintenance of a tight seal, and the bulk of the Hasson cannula has made it less than an ideal trocar. It was these difficulties with the Ilasson technique that prompted our investigation of the newly designed blunt-tipped trocar to gain access and achieve a pneumoperitoneum prior to various urologic laparoscopic procedures.

Although there is a relatively low incidence of major injuries due to the Veress needle and blind trocar placement, the implications in the individual case can be substantial. In the Deziel series, the mortality from bowel injury was 4.6 percent and from vascular injury was 8.8 percent. ${ }^{14}$ Any changes in technique that increase the safety profile without compromising the efficiency of the procedures are certainly welcome. In keeping with this concept, we believe that the trocar discussed in this article has several advantages over other options.

First, the blunt tip of the trocar affords an extra degree of safety in abdominal access. The diameter of the distal blunt end is $3 \mathrm{~mm}$ in diameter, much greater than the obturator of the Veress needle. Since no sharp tip is present, there is an obvious advantage over standard trocars. We are assuming increased safety with these instruments due to the lack of needles and sharp cutting surfaces of the trocar. Certainly, with the limited number of patients in this report, we cannot absolutely verify an increased safety profile. These studies will be forthcoming as more experience is gained with this trocar.

Second, the conical tip requires only a narrow peritoneal opening for insertion. The small opening and the dilating effect of the trocar during insertion create a tight seal of fascia around the trocar to minimize air leaks. Indeed, in our series no air leaks were noted in any patient. This effect 
completely circumvents the need for more costly and complicated designs of trocars, or the use of fascial sutures to prevent air leaks.

Third, the ease of insertion makes this an attractive technique. The mean time to verification of intraperitoneal location with the laparoscope was only two minutes and twenty-nine seconds from the initiation of the skin incision. Insufflation added an additional eighty-nine seconds. Even in individuals with increased weight or increased body mass index, the insertion was equally efficient. Indeed, the shortest insertion time (95 seconds) was in the individual with the highest body mass index (30.1).

There was no correlation between order in the series and insertion time. In other words, insertion time in the first few patients was no different than in the last few patients. This verifies that the insertion technique described in this article can be mastered in a very short period of time.

Why the insertion was in excess of four minutes in 2 patients is unclear because no characteristics of those patients were substantially different from the others in the series, but in each of those cases there was difficulty in stabilizing and elevating the fascia with Kocher clamps. In each case, the skin incision needed to be lengthened slightly to allow proper fixation. If difficulty in clearly identifying the fascia is encountered, our experience would suggest that the skin incision can be extended a few millimeters without compromising the airtight seal at the fascia.

Finally, the cost of the trocar is substantially less than other available models, no doubt due to its simplicity of design. Threads used to secure the trocar to the fascia obviate the need for an additional piece of equipment to achieve this goal, further lowering costs.

We believe that this trocar represents a significant advance in the technique of open laparoscopy due to its apparent safety, simplicity, effectiveness, and cost. We now use this trocar exclusively for access to the abdominal cavity in all urologic laparoscopy cases.
Dand A. Ohl, M.D.

1500 East Medical Center Drive

Ann Arbor, Michigan 48109-03.30

\section{REFERENC.ES}

1. Bloom DA: Two-step orchiopexy with pelviscopic clip ligation of the spermatic vessels. J Unl 145: 1030-1033, 1991.

2. Clayman RV, Kavoussi LR, Soper NI, Dierks SM, Meretyk S, Darcy MD, Roemer FD, Pingleton ED. Thomson PG, and Long SR: I aparoscopic nephrectomy: initial case report. J Urol 146: 278-282, 1991

3. Parra RO, Andrus C, and Boullier J: Staging laparoscopic pelvic lymph node dissection: comparison of results with open pelvic lymphadenectomy. I [ Irol 147: 875-878, 1992.

4. Donovan JF, and Winfield HN: Laparoscopic varix ligation. J Urol 147: 77-81, 1992.

5. Rubenstein SC, Hulbert JC. Pharand D, Schuessler WW, Vancaillie TG, and Kavoussi LR: Laparoscopic ablation of symptomatic cysts. J Urol 150: 1103-1106, 1993.

6. Fahlenkamp D, Raatz D, Schönberger B, and Loening SA: Laparoscopic lymphocele drainage after renal transplantation. J Urol 150: 316-318, 1993.

7. Troxel S, Stanley KE, Winfield HN, and Kreder KJ: Laparoscopic approaches to the treatment of intrinsic urethral weakness (Type III stress urinary incontinence). J Urol 149: 411A, 1993.

8. Zucker KA, Reddick EJ, and Bailey RW: Laparoscopicguided cholecystectomy with electrocautery dissection, in: Zucker KA (Ed): Surgical Laparoscopy, \$1 Louis, Quality Medical Publishing, 1991.

9. Phillips J, Keith D, Hulka J, Hulka B, and Keith L: Gynecologic laparoscopy in 1975. J Reprod Med 16: 105-117, 1976.

10. Yuzpe AA: Pneumoperitoneum needle and trocar injuries in laparoscopy a survey on possible contributing factors and prevention. J Reprod Med 35: 485-490, 1990.

11. Riedel HH, Lehmann-Willenbrock L, Conrad P, and Semm K: German pelviscopic statistics for the years 1978-1982. Endoscopy 18: 219-222, 1986.

12. Capelouto CC, and Kavoussi L.R: Complications of laparoscopic surgery. Urology 42: 2-12, 1993.

13. Lehmann-Willenbrock $E$, Riedel $H H$, Mecke $H$, and Semm K: Pelviscopy/laparoscupy and ils complications in Germany 1949-1988. J Reprod Med 37: 671-677, 1992.

14. Deziel DJ, Millikan KW, Economou SG, Doolas A, Ko ST, and Airan MC: Complications of laparoscopic cholecystectomy: a national survey of 4,292 hospitals and an analysis of 77,604 cases. Am J Surg 165: 9- 14, 1993.

15. Hasson HM: A modified instrument and method for laparoscopy. Am J Obstet Gynecol 110: 886-887, 1971. 